Check for updates

Cite this: RSC Adv., 2017, 7, 46894

Received 3rd June 2017

Accepted 20th August 2017

DOI: $10.1039 / \mathrm{c} 7 \mathrm{ra06183h}$

rsc.li/rsc-advances

\section{Kinetic study of application of graphene oxide as a catalyst to accelerate extraction of total flavonoids from Radix Scutellaria}

\begin{abstract}
Xin Wang (D) *ab and Fengyu Wei
A new kinetic model, So's three-step kinetic model, was used to study the kinetics of graphene oxide (GO)assisted ethanol reflux extraction (GERE). An experimental design to investigate the effect of five single factors (extraction temperature, dosage amount of GO, concentration of ethanol aqueous solution, the ratio of liquid to solid, rotating speed) on the kinetic extraction of total flavonoids was materialized. From the single factors experiments, we found that the extraction process is mainly controlled by internal diffusion, and the extraction rate increases with the growth of GO dosage, ethanol concentration and liquid-solid ratio at 30-70 ${ }^{\circ} \mathrm{C}$; moreover, the rotating speed had little effect. The effective internal diffusion coefficient $\left(D^{\prime}\right)$ and the activation energy $\left(E_{\mathrm{a}}\right)$ of total flavonoids extracted by ERE and GERE were also studied, respectively. Results showed a good prediction of So's three-step kinetic model for extraction kinetics in all experiments, which gave the possibility for estimating the initial rate and extent of GERE.
\end{abstract}

\section{Introduction}

Radix Scutellaria is a commonly used herbal medicine that is extensively used for the treatment of inflammation, pyrexia, jaundice, diarrhea and hepatitis, etc. ${ }^{\mathbf{1 , 2}}$ The major types of flavonoids in Radix Scutellaria are baicalin (BG), baicalein (B), wogonoside (WG), and wogonin (W). ${ }^{3}$ The pharmacological studies in vivo and in vitro have shown that $\mathrm{B}$ and $\mathrm{W}$ exhibit stronger bioactivities than their glucuronides BG and WG, particularly with respect to their antioxidant and antiangiogenesis properties. ${ }^{4,5}$ Scutellaria baicalensis flavonoids have antibacterial, antifungal, anti-cancer, anti-virus, antimicrobial, anti-inflammatory and anti-metamorphosis, antioxidant and other biological effects. ${ }^{1}$ At present, the methods of extracting flavonoids from Scutellaria baicalensis are mainly the solvent extraction method, ${ }^{6}$ ultrasonic extraction method, ${ }^{7}$ acid hydrolysis method and enzymatic hydrolysis method. ${ }^{8,9}$ The acid hydrolysis method and the enzymatic hydrolysis method are currently recognized as novel and superior extraction methods, but problems still exist. The former is often restricted by the relatively high cost of highly efficient enzymes and the latter often involve hydrochloric acid or sulfuric acid as a catalyst, bringing a large amount of acidic effluent that may damage the environment. Therefore, it is necessary to develop a novel green process to resolve the problems mentioned above. In a previous

${ }^{a}$ School of Petrochemical Technology, JiLin Institute of Chemical Technology, JiLin 132022, P. R. China. E-mail: wangxin1990090@126.com; Fax: +86 15144206765; Tel: +8615144206765

${ }^{b}$ School of Chemical Engineering, Hefei University of Technology, Hefei 230009, P. R. China study, ${ }^{10}$ we, for the first time, used graphene oxide (GO) to catalyze the hydrolyzation of Scutellaria flavone glycosides and supplied an alternative approach to green hydrolyzation, extending the application of GO. It was found that the GOassisted ethanol reflux extraction possesses a synergistic effect between the GO hydrolysis of cell walls and the flavonoids leaching in the ethanol aqueous solution, which significantly increased the extraction rate of the total flavonoids and baicalein. ${ }^{10}$ For industrial applications and mass production, the kinetics study is necessary, because the kinetics study of GERE can give a good prediction for the extraction kinetics in experiments, which gives the possibility for estimating the initial rate and extent of GERE. Research on GERE extraction kinetics has not been reported. Herein, we used a new kinetics model, So's three-step kinetic model, ${ }^{11}$ to study the kinetics mechanism of GERE, in order to establish the extraction kinetics equation, which gives the possibility for estimating the initial rate and extent of GO-assisted ethanol reflux extraction (GERE). To achieve these goals, we studied the influence of extraction time, extraction temperature, ethanol concentration, GO's dosage, rotating speed and ratio of solvent to raw material on the extraction yields of the total flavonoids. The conventional ethanol reflux extraction method (ERE) was also carried out and treated as a control.

\section{Materials and methods}

\subsection{Materials}

Scutellaria radix pieces (Inner Mongolia Scutellaria) were purchased from Hefei Pharmacies CO., China. All Scutellaria radix pieces were cleaned, separated from impurities, dried and 
sieved to 10-20 mesh for the following experiments. GO was prepared by a modified Hummers method. ${ }^{12}$ The specific prepared process and the characterization results of GO can be seen in our earlier studies. ${ }^{10}$

\subsection{Chemicals and reagents}

The flavone standards, baicalin (98\%) and baicalein (98\%) were purchased from Chinese Food and Drug Testing Institute (Shanghai, China). Ethanol (99.7\%) of analytical grade for extraction was bought from Sinopharm Chemical Reagent Co. (Shanghai, China). Deionized water was prepared using a water purification system purchased from HITECH Instruments Co. LTD (Henan, China).

\subsection{Extraction procedures}

2.3.1 Extraction devices. The $\mathrm{HH}-2$ digital thermostat water bath was purchased from Guohua Electric Co. (Shanghai, China). A UV-2550 UV-visible spectrophotometer was purchased from Shimadzu Corporation (Tokyo, Japan).

2.3.2 GO-assisted ethanol reflux extraction (GERE). Radix Scutellaria powder $(10.0 \mathrm{~g})$ was loaded in a $250 \mathrm{~mL}$ flask (flat bottom) containing a volume of extraction solvent and an amount of GO according to the experimental design. The flask was then immersed into the digital thermostat water bath to maintain a constant temperature throughout the period of extraction. The temperature of the flask was measured with a mercury-in-glass thermometer having $\pm 0.1{ }^{\circ} \mathrm{C}$ divisions. The extraction experiments were started when the system reached the desired temperature. To prevent the loss of solvent during extraction, a condenser was connected to the flask. Using the experimental design, the effect of five single factors (extraction temperature, dosage amount of GO, concentration of ethanol aqueous solution, the ratio of liquid to solid, rotating speed) on the yields of total flavonoids $(\eta)$ was investigated under the extraction process, and samples were taken at 1, 2, 3, 4, 5, 10, 20, $30,40,50,60,80,100,120,150,180,240,300$, and $360 \mathrm{~min}$. After extraction, the resulting solution was centrifuged, and the supernatant was decanted away for UV analysis. At least two replicates were performed for each extraction condition tested, and all analyses were repeated three times. The yields of total flavonoids $(\eta)$ was defined as follows:

$$
\eta=\frac{C \times V}{1000 m} \times 100 \%
$$

$C$ was the total flavonoids' concentration of the extraction solution $\left(\mathrm{mg} \mathrm{mL}{ }^{-1}\right)$, calculated by the standard curve detected by UV-visible spectrophotometry, ${ }^{13} V$ was the extraction solution volume (mL), $m$ was the quality of dry raw material $(\mathrm{g})$. Simultaneously, ERE was also performed without GO as a catalyst at the same experimental conditions compared with GERE.

2.3.3 Modeling equations. The extraction of total flavonoids from Scutellaria was assumed to be a three-step extraction. First, the solvent dissolves the solute and penetrates into the Scutellaria cells. Second, the flavonoid particles diffuse from the surface of the Scutellaria cells (inner diffusion), and then finally diffuse into the solvent (external diffusion). External diffusion resistance can be ignored because of extensive stirring during the extraction process. So et al. ${ }^{11}$ referred to the three stages of these two processes as the washing stage, the first diffusion stage (fast diffusion stage) and the second diffusion stage (slow diffusion stage). So et al. ${ }^{11}$ described the three-stage model according to Fick's second law, and the extraction rate of the ingredient product can be expressed by the equation:

$$
\begin{aligned}
\eta_{t}= & \eta_{\mathrm{e}}^{\mathrm{w}}\left[1-\exp \left(-k_{\mathrm{w}} t\right)\right]+\eta_{\mathrm{e}}^{\mathrm{d} 1}\left[1-\exp \left(-k_{\mathrm{d} 1} t\right)\right] \\
& +\eta_{\mathrm{e}}^{\mathrm{d} 2}\left[1-\exp \left(-k_{\mathrm{d} 2} t\right)\right]
\end{aligned}
$$

Generally, the washing stage is extremely fast and $k_{\mathrm{w}}$ is very large, ${ }^{\mathbf{1 4}}$ and hence the $\exp \left(-k_{\mathrm{w}} t\right)$ can be ignored and eqn (2) can be simplified as follows:

$$
\eta_{t}=\eta_{\mathrm{e}}^{\mathrm{w}}+\eta_{\mathrm{e}}^{\mathrm{d} 1}\left[1-\exp \left(-k_{\mathrm{d} 1} t\right)\right]+\eta_{\mathrm{e}}^{\mathrm{d} 2}\left[1-\exp \left(-k_{\mathrm{d} 2} t\right)\right]
$$

The extraction rate of flavonoids can be expressed by the equation:

$$
\begin{gathered}
\eta_{\mathrm{e}}=\eta_{\mathrm{e}}^{\mathrm{w}}+\eta_{\mathrm{e}}^{\mathrm{d} 1}+\eta_{\mathrm{e}}^{\mathrm{d} 2} \\
\eta_{\mathrm{e}}-\eta_{t}=\eta_{\mathrm{e}}^{\mathrm{d} 1} \exp \left(-k_{\mathrm{d} 1} t\right)+\eta_{\mathrm{e}}^{\mathrm{d} 2} \exp \left(-k_{\mathrm{d} 2} t\right)
\end{gathered}
$$

Eqn (5) is applied to fit the experimental data. $\eta_{\mathrm{e}}$, which is the equilibrium extraction rate of Scutellaria flavonoids, can be measured experimentally. The equation parameters $k_{\mathrm{d} 1}, k_{\mathrm{d} 2}$, $\eta_{\mathrm{e}}^{\mathrm{d} 1}$ and $\eta_{\mathrm{e}}^{\mathrm{d} 2}$ can be obtained by fitting with $t$ and $\left(\eta_{\mathrm{e}}-\eta_{t}\right)$ by origin 8.0.

\section{Results and discussion}

\subsection{Kinetics of Scutellaria flavonoids extraction}

3.1.1 Effect of extraction temperature. First, to study the effect of extraction temperature on GERE and ERE extraction kinetics, the extraction temperature was varied from $30{ }^{\circ} \mathrm{C}$ to $70{ }^{\circ} \mathrm{C}$ on the rotational speed $270 \mathrm{rpm}$, and the samples were taken at 1, 2, 3, 4, 5, 10, 20, 30, 40, 50, 60, 80, 100, 120, 150, 180, 240,300 , and $360 \mathrm{~min}$. The other parameters of ERE and GERE were set as the optimal conditions based on our previous investigations. ${ }^{10}$ Table 1 shows the results of experimental data fitted with eqn (5).

As can be seen from Table 1, for both ERE and GERE, the $R^{2}$ is above 0.99 and the $\mathrm{Chi}^{\wedge} 2 / \mathrm{DoF}$ is below $0.02 \%$, indicating that the three-stage model derived from the Fick's second law feasibly describes the extraction rate of Scutellaria flavonoids. Irrespective of ERE or GERE, $\eta_{\mathrm{e}}^{\mathrm{d} 1}$ is much larger than $\eta_{\mathrm{e}}^{\mathrm{d} 2}$, indicating that the extraction of total flavonoids of Scutellaria is mainly done in the fast diffusion stage. The results indicated that $k_{\mathrm{d} 1}, k_{\mathrm{d} 2}, \eta_{\mathrm{e}}^{\mathrm{d} 1}$ and $\eta_{\mathrm{e}}^{\mathrm{d} 2}$ by ERE and GERE increased with temperature growth. This is, on one hand, because temperature growth increases the speed of molecular diffusion, enables the increase of permeate, and promotes the diffusion and dissolution of flavonoids. On the other hand, Fick's law shows that the extraction temperature is proportional to the rate of extraction, when the temperature is increased, the solvent viscosity is reduced, which leads to reduced resistance by $\mathrm{Scu}$ tellaria flavonoids through the solid-liquid interface. This is 
Table 1 Effect of temperature on the kinetic extraction of flavonoids from Scutellaria

\begin{tabular}{|c|c|c|c|c|c|c|c|c|c|c|c|c|c|c|}
\hline \multirow[b]{2}{*}{$T /{ }^{\circ} \mathrm{C}$} & \multicolumn{7}{|l|}{ ERE } & \multicolumn{7}{|l|}{ GERE } \\
\hline & $\begin{array}{l}k_{\mathrm{d} 1} \times \\
10^{2} / \min ^{-1}\end{array}$ & $\begin{array}{l}k_{\mathrm{d} 2} \times \\
10^{2} / \mathrm{min}^{-1}\end{array}$ & $\eta_{\mathrm{e}}^{\mathrm{d} 1} / \%$ & $\eta_{\mathrm{e}}^{\mathrm{d} 2} / \%$ & $y_{0}$ & $R^{2}$ & $\begin{array}{l}\mathrm{Chi}^{\wedge} 2 / \\
\mathrm{DoF} \times 10^{4}\end{array}$ & $\begin{array}{l}k_{\mathrm{d} 1} \times \\
10^{2} / \mathrm{min}^{-1}\end{array}$ & $\begin{array}{l}k_{\mathrm{d} 2} \times \\
10^{2} / \mathrm{min}^{-1}\end{array}$ & $\eta_{\mathrm{e}}^{\mathrm{d} 1} / \%$ & $\eta_{\mathrm{e}}^{\mathrm{d} 2} / \%$ & $y_{0}$ & $R^{2}$ & $\begin{array}{l}\mathrm{Chi}^{\wedge} 2 / \\
\mathrm{DoF} \times 10^{4}\end{array}$ \\
\hline 30 & 9.86 & 0.53 & 68.6 & 9.8 & -0.012 & 0.999 & 0.29 & 11.42 & 1 & 70.8 & 10.5 & -0.0024 & 0.999 & 0.77 \\
\hline 40 & 9.98 & 0.54 & 68.9 & 9.9 & -0.0109 & 0.999 & 0.31 & 11.55 & 1.03 & 71.4 & 10.8 & 0.0033 & 0.999 & 0.94 \\
\hline 50 & 10.12 & 0.56 & 69.2 & 9.96 & -0.0095 & 0.999 & 0.33 & 12.88 & 1.08 & 73.6 & 11.6 & -0.0197 & 0.999 & 1.95 \\
\hline 60 & 10.53 & 0.72 & 69.5 & 10.1 & -0.0038 & 0.999 & 0.38 & 13.6 & 1.11 & 75.9 & 12 & 0.00398 & 0.999 & 0.99 \\
\hline 70 & 11.32 & 0.96 & 70.9 & 10.3 & 0.00154 & 0.999 & 0.73 & 13.9 & 1.12 & 76.4 & 12.01 & 0.00368 & 0.999 & 1.25 \\
\hline
\end{tabular}

due to the growth of the mass transfer rate. This is consistent with the result that Mezuane et $a l .{ }^{15}$ found in the ethanol reflux extraction of olive oil that both $k_{\mathrm{d} 1}$ and $\eta_{\mathrm{e}}^{\mathrm{d} 1}$ increase with temperature increasing. $k_{\mathrm{d} 1}$ was $9.86 \times 10^{-2}$ to $13.9 \times$ $10^{-2} \mathrm{~min}^{-1}$ at $30-70{ }^{\circ} \mathrm{C}$, similar to that reported in literature ${ }^{16}$ by ultrasonic extraction of total flavonoids from Prunella vulgaris. From Table 1 , it is clear that $k_{\mathrm{d} 1}, k_{\mathrm{d} 2}, \eta_{\mathrm{e}}^{\mathrm{d} 1}$ and $\eta_{\mathrm{e}}^{\mathrm{d} 2}$ by GERE are significantly higher than that by ERE. It may be attributed to GO destruction of the structure of the cell wall, ${ }^{10,13}$ which reduces the resistance of the diffusion phase and accelerates both the fast diffusion stage and the slow diffusion stage.

3.1.2 Effect of ethanol concentration. The experiments studying the effect of ethanol concentration on the extraction kinetics were performed at a ratio of solvent to raw material of $15\left(\mathrm{~mL} \mathrm{~g}^{-1}\right)$, an extraction temperature of $70{ }^{\circ} \mathrm{C}$, and a dosage of GO for GERE of $1.1 \mathrm{mg} \mathrm{g}^{-1} .^{10}$ The results are shown in Table 2. As can be seen from Table 2, for both ERE and GERE, the $k_{\mathrm{d} 1}$, $k_{\mathrm{d} 2}, \eta_{\mathrm{e}}^{\mathrm{d} 1}$ and $\eta_{\mathrm{e}}^{\mathrm{d} 2}$ increased with the growth in concentration of aqueous ethanol to the maximum at $60 \%$ and then decreased.

The increase in the extraction ability of total flavonoids with the introduction of ethanol to water can be attributed to the increase in permeability of plant tissues, which enables better mass transfer by diffusion. Previous literature ${ }^{17,18}$ also reported that the optimal ethanol concentration in the extraction of flavonoids from Radix Scutellariae is about 40-75\%. When ethanol aqueous solution has similar polarity with Scutellaria's flavonoids, the rate of total flavonoids is higher according to the "like dissolves like" principle. Therefore, it is reasonable that the optimum concentration of ethanol is $60 \%$. From Table 2, the $k_{\mathrm{d} 1}, k_{\mathrm{d} 2}, \eta_{\mathrm{e}}^{\mathrm{d} 1}$ and $\eta_{\mathrm{e}}^{\mathrm{d} 2}$ by GERE at each alcohol concentration are higher than that by ERE, because GO has a synergistic effect on the hydrolysis of cell walls and the leaching of flavonoids with an ethanol solution, which effectively reduces the mass transfer resistance and enables better mass transfer by diffusion.

3.1.3 Effect of GO dosage. The dosage of GO is a key parameter that decides the activity of the catalytic reaction and influences the extraction kinetics. Table 3 shows the effect of GO dosage on the kinetics of Scutellaria flavonoids extraction at $70{ }^{\circ} \mathrm{C}$.

As seen from Table 3 , the $k_{\mathrm{d} 1}, k_{\mathrm{d} 2}, \eta_{\mathrm{e}}^{\mathrm{d} 1}$ and $\eta_{\mathrm{e}}^{\mathrm{d} 2}$ first increased with an increase in the GO dosage $(\mathrm{g})$, achieving a maximum at $1.1 \mathrm{mg} \mathrm{g}^{-1}$ and then decreased. Moreover, the acidity of the solvent was significantly enhanced with the addition of GO. ${ }^{\mathbf{1 0}}$ This is because the addition of GO, on one hand, destroys the cell wall structure, and has a synergistic effect with the ethanol solution; on the other hand, it may lead to more baicalin decomposing into baicalein..$^{\mathbf{1 , 3 , 1 0}}$ Therefore, when the dosage of GO is less than $1.1 \mathrm{mg} \mathrm{g}^{-1}$, the destruction of the cell wall is enhanced with increasing amounts of GO, resulting in reduced resistance of extraction and the growth of the mass transfer rate, thus increasing $k_{\mathrm{d} 1}, k_{\mathrm{d} 2}, \eta_{\mathrm{e}}^{\mathrm{d} 1}$ and $\eta_{\mathrm{e}}^{\mathrm{d} 2}$. When the amount of added GO exceeded $1.1 \mathrm{mg} \mathrm{g}^{-1}$, the dosage of GO increased baicalin decomposing into baicalein and a lot of glucose, which enhanced the resistance of the flavonoids diffusing to the solvent and decreased the mass transfer rate, thus reducing $k_{\mathrm{d} 1}$, $k_{\mathrm{d} 2}, \eta_{\mathrm{e}}^{\mathrm{d} 1}$ and $\eta_{\mathrm{e}}^{\mathrm{d} 2}$. Moreover, as can be observed from the curve between the dosage of GO and pH shown in Fig. 1, the acidity of the solvent is obviously enhanced with the addition of GO. The higher acidity of solvent leads to more baicalin decomposing into baicalein, and hence the yields of total flavonoids decline. It has been reported that baicalin and baicalein are very unstable in strong acidic environment, ${ }^{1,3}$ which may also cause a decline in the yields of total flavonoids.

3.1.4 Effect of the ratio of solvent to raw material. The effect of the ratio of solvent to raw material $\mathrm{S}\left(\mathrm{mL} \mathrm{g}^{-1}\right)$ on the

Table 2 Effect of ethanol concentration on the kinetic extraction of flavonoids from Scutellaria

\begin{tabular}{|c|c|c|c|c|c|c|c|c|c|c|c|c|c|c|}
\hline \multirow[b]{2}{*}{$C / \%$} & \multicolumn{7}{|l|}{ ERE } & \multicolumn{7}{|l|}{ GERE } \\
\hline & $\begin{array}{l}k_{\mathrm{d} 1} \times \\
10^{2} / \min ^{-1}\end{array}$ & $\begin{array}{l}k_{\mathrm{d} 2} \times \\
10^{2} / \min ^{-1}\end{array}$ & $\eta_{\mathrm{e}}^{\mathrm{d} 1} / \%$ & $\eta_{\mathrm{e}}^{\mathrm{d} 2} / \%$ & $y_{0}$ & $R^{2}$ & $\begin{array}{l}\mathrm{Chi}^{\wedge} 2 / \mathrm{DoF} \\
\times 10^{4}\end{array}$ & $\begin{array}{l}k_{\mathrm{d} 1} \times \\
10^{2} / \min ^{-1}\end{array}$ & $\begin{array}{l}k_{\mathrm{d} 2} \times \\
10^{2} / \min ^{-1}\end{array}$ & $\eta_{\mathrm{e}}^{\mathrm{d} 1} / \%$ & $\eta_{\mathrm{e}}^{\mathrm{d} 2} / \%$ & $y_{0}$ & $R^{2}$ & $\begin{array}{l}\mathrm{Chi}^{\wedge} 2 / \mathrm{DoF} \\
\times 10^{4}\end{array}$ \\
\hline 40 & 10.48 & 0.65 & 69.7 & 10.05 & -0.00501 & 0.999 & 0.43 & 10.8 & 0.67 & 71.5 & 9.79 & -0.0048 & 0.999 & 0.46 \\
\hline 50 & 10.88 & 0.84 & 69.8 & 10.12 & 0.00147 & 0.999 & 0.56 & 13.6 & 1.07 & 75.6 & 12.22 & 0.0045 & 0.999 & 0.94 \\
\hline 60 & 11.32 & 0.96 & 70.9 & 10.3 & 0.00154 & 0.999 & 0.73 & 13.9 & 1.12 & 76.4 & 12.01 & 0.00368 & 0.999 & 1.25 \\
\hline 70 & 11.01 & 0.94 & 70.3 & 9.92 & 0.00702 & 0.999 & 0.59 & 12.01 & 1.09 & 71.8 & 10.66 & 0.00324 & 0.999 & 0.9 \\
\hline
\end{tabular}


Table 3 Effect of dosage of GO on the kinetic extraction of flavonoids from Scutellaria

\begin{tabular}{|c|c|c|c|c|c|c|c|}
\hline $\begin{array}{l}\mathrm{GO} / \mathrm{mg} \\
\mathrm{g}^{-1}\end{array}$ & $k_{\mathrm{d} 1} \times 10^{2} / \mathrm{min}^{-1}$ & $k_{\mathrm{d} 2} \times 10^{2} / \mathrm{min}^{-1}$ & $\eta_{\mathrm{e}}^{\mathrm{d} 1} / \%$ & $\eta_{\mathrm{e}}^{\mathrm{d} 2} / \%$ & $y_{0}$ & $R^{2}$ & $\begin{array}{l}\mathrm{Chi}^{\wedge} 2 / \mathrm{DoF} \\
\times 10^{4}\end{array}$ \\
\hline 0 & 11.32 & 0.96 & 70.9 & 10.3 & 0.00154 & 0.999 & 0.73 \\
\hline 0.55 & 13.34 & 0.97 & 72.8 & 11.6 & 0.0031 & 0.999 & 7.36 \\
\hline 1.1 & 13.9 & 1.12 & 76.4 & 12.01 & 0.00368 & 0.999 & 1.25 \\
\hline 2.2 & 10.86 & 0.68 & 71.56 & 9.86 & -0.00428 & 0.999 & 4.84 \\
\hline
\end{tabular}

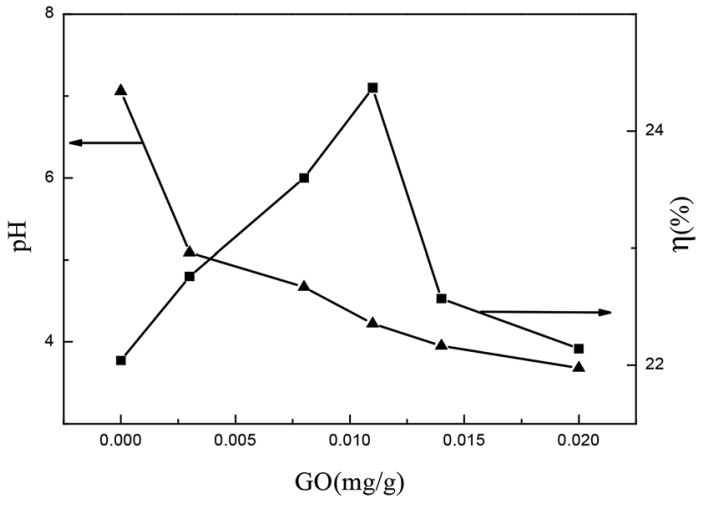

Fig. $1 \mathrm{pH}$ and yields of total flavonoids under different GO dosages.

extraction kinetics is shown in Table 4 . The experiments were carried out in the above optimization conditions.

From Table 4 , it can be seen that $k_{\mathrm{d} 1}, k_{\mathrm{d} 2}, \eta_{\mathrm{e}}^{\mathrm{d} 1}$ and $\eta_{\mathrm{e}}^{\mathrm{d} 2}$ by ERE increase with the growth of liquid/solid ratio. This is because the growth of $\mathrm{S}$ increases the mass transfer interfacial area and mass transfer rate. ${ }^{19}$ However, for GERE, $k_{\mathrm{d} 1}, k_{\mathrm{d} 2}, \eta_{\mathrm{e}}^{\mathrm{d} 1}$ and $\eta_{\mathrm{e}}^{\mathrm{d} 2}$ first increased with the growth of the liquid/solid ratio up to the maximum at $15\left(\mathrm{~mL} \mathrm{~g}^{-1}\right)$ and then decreased. This is because GO's concentration was reduced when the ratio of solvent to raw material increased, which weakened its catalytic activity and reduced the mass transfer rate.

3.1.5 Effect of the rotating speed. The effect of rotating speed on the extraction kinetics was investigated at rotating speeds ranging between 180 and $350 \mathrm{rpm}$. The results of the rotating speed experiments are shown in Table 5.

Generally, ${ }^{20}$ the rotating speed affects the extraction process, which is controlled only by diffusion or both diffusion and chemical reaction. However, for the extraction process controlled by the internal reaction, the extraction rate has nothing to do with the rotating speed. Table 5 shows that irrespective of ERE or GERE $k_{\mathrm{d} 1}, k_{\mathrm{d} 2}, \eta_{\mathrm{e}}^{\mathrm{d} 1}$ and $\eta_{\mathrm{e}}^{\mathrm{d} 2}$ show little change with rotating speed, which means that rotating speed has little effect on the extraction kinetics, further indicating that the extraction process of total flavonoids is controlled by the inner diffusion.

\subsection{Apparent diffusion coefficient $\left(D^{\prime}\right)$ and the activation energy $\left(E_{\mathrm{a}}\right)$}

As seen, the Scutellaria particles sieved to 10-20 mesh are not spherical but cylindrical, and it can be assumed that the apparent diffusion coefficient of each stage on the extraction process is consistent with the apparent diffusion coefficient of Fick's second law based on the model proposed by Kang et al. ${ }^{21}$

$$
D^{\prime}=D / \lambda^{2}=\frac{k R_{0}^{2}}{5.78}
$$

The extraction of $D^{\prime}$ by Scutellaria's total flavonoids can be calculated according to Table 1 and eqn (6), and the results are shown in Table 6. From Table 6, it can be observed that the value of $D^{\prime}$ is in the range $3.57-5.03 \times 10^{-12} /\left(\mathrm{m}^{2} \mathrm{~s}^{-1}\right)$. The ADC from GERE has not been reported in the literature. Zhao et al. ${ }^{22}$ reported that the ADC of polysaccharides ranged from $10^{-12}$ to $10^{-11} /\left(\mathrm{m}^{2} \mathrm{~s}^{-1}\right)$, which is similar to our experimental data. Generally, ${ }^{20}$ the ADC $(D)$ of rhizome is about $10^{-11} /\left(\mathrm{m}^{2} \mathrm{~s}^{-1}\right)$, for example, the $D$ of glycyrrhizic acid extracted by the aqueous ammonia method from licorice root was $5.1 \times 10^{-11} /\left(\mathrm{m}^{2} \mathrm{~s}^{-1}\right)$, the $D$ of valerian in valerian root extracted by ethanol was $0.82 \times 10^{-11} /\left(\mathrm{m}^{2} \mathrm{~s}^{-1}\right)$.

As we know, $D^{\prime}$ is related to not only temperature but also the particle size. It is assumed that the correction coefficient $\lambda$ of the mass transfer path is independent of temperature, and the relationship between $\lambda$ and $R_{0}$ is $\lambda=m R_{0}{ }^{-n}{ }^{23}$ The mass transfer path of GERE changes because GO damage to the cell wall of

Table 4 Effect of liquid-solid ratio on the kinetic extraction of flavonoids from Scutellaria

\begin{tabular}{|c|c|c|c|c|c|c|c|c|c|c|c|c|c|c|}
\hline $\mathrm{S} / \mathrm{mL} \mathrm{g}^{-1}$ & \multicolumn{7}{|l|}{ ERE } & \multicolumn{7}{|l|}{ GERE } \\
\hline 10 & 10.25 & 0.57 & 69.6 & 10.1 & -0.0067 & 0.999 & 0.45 & 12.9 & 0.85 & 69.9 & 10.5 & -0.0329 & 0.999 & 2.27 \\
\hline 12 & 10.46 & 0.66 & 69.9 & 10.2 & -0.0050 & 0.999 & 0.45 & 13.7 & 0.92 & 71.9 & 11.74 & 0.00281 & 0.999 & 0.85 \\
\hline 15 & 11.32 & 0.96 & 70.9 & 10.3 & 0.00154 & 0.999 & 0.73 & 13.9 & 1.12 & 76.4 & 12.01 & 0.00368 & 0.999 & 1.25 \\
\hline
\end{tabular}


Table 5 Effect of rotational speed on the kinetic extraction of flavonoids from Scutellaria

\begin{tabular}{|c|c|c|c|c|c|c|c|c|c|c|c|c|c|c|}
\hline \multirow[b]{2}{*}{$n / \mathrm{rpm}$} & \multicolumn{7}{|l|}{ ERE } & \multicolumn{7}{|l|}{ GERE } \\
\hline & $\begin{array}{l}k_{\mathrm{d} 1} \times \\
10^{2} / \mathrm{min}^{-1}\end{array}$ & $\begin{array}{l}k_{\mathrm{d} 2} \times \\
10^{2} / \min ^{-1}\end{array}$ & $\eta_{\mathrm{e}}^{\mathrm{d} 1} / \%$ & $\eta_{\mathrm{e}}^{\mathrm{d} 2} / \%$ & $y_{0}$ & $R^{2}$ & $\begin{array}{l}\mathrm{Chi}^{\wedge} 2 / \mathrm{DoF} \\
\times 10^{4}\end{array}$ & $\begin{array}{l}k_{\mathrm{d} 1} \times \\
10^{2} / \mathrm{min}^{-1}\end{array}$ & $\begin{array}{l}k_{\mathrm{d} 2} \times \\
10^{2} / \min ^{-1}\end{array}$ & $\eta_{\mathrm{e}}^{\mathrm{d} 1} / \%$ & $\eta_{\mathrm{e}}^{\mathrm{d} 2} / \%$ & $y_{0}$ & $R^{2}$ & $\begin{array}{l}\mathrm{Chi}^{\wedge} 2 / \mathrm{DoF} \\
\times 10^{4}\end{array}$ \\
\hline 180 & 10.35 & 0.65 & 70.41 & 9.88 & -0.0087 & 0.999 & 1.07 & 12.9 & 1.02 & 72.4 & 14.8 & 0.00346 & 0.999 & 0.36 \\
\hline 270 & 11.32 & 0.96 & 70.9 & 10.3 & 0.00154 & 0.999 & 0.73 & 13.9 & 1.12 & 76.4 & 12.01 & 0.00368 & 0.999 & 1.25 \\
\hline 350 & 11.44 & 0.98 & 70.95 & 10.55 & 0.00193 & 0.999 & 0.82 & 13.67 & 1.27 & 76.3 & 12.1 & 0.0052 & 0.999 & 0.94 \\
\hline
\end{tabular}

Table 6 Expansive diffusion coefficient in rapid diffusion stage $D^{\prime} \times$ $10^{12} /\left(\mathrm{m}^{2} \mathrm{~s}^{-1}\right)$

\begin{tabular}{lll}
\hline$T /{ }^{\circ} \mathrm{C}$ & ERE & GERE \\
\hline 30 & 3.57 & 4.13 \\
40 & 3.61 & 4.18 \\
50 & 3.66 & 4.66 \\
60 & 3.81 & 4.92 \\
70 & 4.09 & 5.03
\end{tabular}

Scutellaria changes the cell permeability. The effect of GO depends on temperature, ${ }^{\mathbf{8} 10,12}$ and hence $\lambda$ is not only related to $R_{0}$, but also related to temperature. Therefore, it can be assumed that the relationship between $\lambda$ and temperature and particle size is

$$
\lambda=\left(m R_{0}{ }^{-n}\right) /\left(T-T_{\mathrm{c}}\right)^{2 \theta}
$$

The relationship between the diffusion coefficient $D$ and the extraction temperature follows the Arrhenius formula

$$
D=A \exp \left(-\frac{E_{\mathrm{a}}}{R T}\right)
$$

Hence, the diffusion coefficient $D^{\prime}$ of ERE and GERE can be obtained as follows:

$$
\mathrm{ERE}: D^{\prime}=D / \lambda^{2}=\left\{A \exp \left(-\frac{E_{\mathrm{a}}}{R T}\right)\right\} /\left(m R_{0}{ }^{-n}\right)^{2}
$$

GERE : $D^{\prime}=D / \lambda^{2}$

$$
=\left\{A \exp \left(-\frac{E_{\mathrm{a}}}{R T}\right)\right\} /\left\{\left(m R_{0}{ }^{-n}\right) /\left(T-T_{\mathrm{c}}\right)^{2 \theta}\right\}^{2}
$$

Take the logarithm on both sides of (9) and (10)

$$
\begin{gathered}
\text { ERE: } \ln D^{\prime}=\ln \left(A / m^{2}\right)-E_{\mathrm{a}} /(R T)+2 n \ln R_{0} \\
\text { GERE: } \begin{aligned}
\ln D^{\prime}= & \ln \left(A / m^{2}\right)-E_{\mathrm{a}} /(R T)+2 n \ln R_{0} \\
& +2 \theta \ln \left(T-T_{\mathrm{c}}\right)^{2}
\end{aligned}
\end{gathered}
$$

$\ln D^{\prime}$ and $1 / T$ were linearly analyzed according to the data of Table 6 and the formulas (11) and (12), and the relationship between $D^{\prime}$ and $T$ was obtained as follows when ERE was extracted at $30-70{ }^{\circ} \mathrm{C}$ :

$$
\ln D^{\prime}=\ln \left(A / m^{2}\right)-2.45 / T+2 n \ln R_{0}
$$

For GERE (the dosage of GO is $1.1 \mathrm{mg} \mathrm{g}^{-1}$ ), the fitting equation is

$$
\ln D^{\prime}=\ln \left(A / m^{2}\right)-1.69 / T+2 n \ln R_{0}+2 \theta \ln \left(T-T_{\mathrm{c}}\right)^{2}
$$

Calculated from the above two formulas, $E_{\mathrm{a}}$ was $20.37 \mathrm{~kJ} \mathrm{~mol}^{-1}$ for ERE and $13.2 \mathrm{~kJ} \mathrm{~mol}^{-1}$ for GERE. This further indicated that the addition of GO greatly accelerates the progress of the reaction.

\section{Conclusion}

The extraction process of total flavonoids from Scutellaria baicalensis is well described by a three-stage model based on Fick's second law. The extraction rate increased with the growth of GO dosage, ethanol concentration and liquid-solid ratio at $30-70{ }^{\circ} \mathrm{C}$, and the rotating speed had little effect. This result is highly consistent with our previous study on single factor experiments. ${ }^{\mathbf{1 0}}$ In this study, the effective internal diffusion coefficient $D^{\prime}$ of flavonoids extracted by ERE and GERE was studied. The results showed that, for ERE, $\ln D^{\prime}=\ln \left(A / m^{2}\right)-2.45 / T+2 n \ln R_{0}$ and $E_{\text {a }}$ $=20.37 \mathrm{~kJ} \mathrm{~mol}^{-1}$, and for GERE, $\ln D^{\prime}=\ln \left(A / m^{2}\right)-1.69 / T+$ $2 n \ln R_{0}+2 \theta \ln \left(T-T_{\mathrm{c}}\right)^{2}$ and $E_{\mathrm{a}}=13.2 \mathrm{~kJ} \mathrm{~mol}^{-1}$, which was $35.2 \%$ lower than that by ERE. At $30-70{ }^{\circ} \mathrm{C}$, the apparent diffusion coefficient $\left(D^{\prime}\right)$ of GERE extraction is $4.13-5.03 \times 10^{-12} /\left(\mathrm{m}^{2} \mathrm{~s}^{-1}\right)$, and the $D^{\prime}$ of ERE is $3.57-4.09 \times 10^{-12} /\left(\mathrm{m}^{2} \mathrm{~s}^{-1}\right)$.

\section{Conflicts of interest}

There are no conflicts to declare.

\section{References}

1 H. B. Li, Y. Jiang and F. Chen, Separation methods used for Scutellaria baicalensis active components, J. Chromatogr. B: Anal. Technol. Biomed. Life Sci., 2004, 812, 277-290.

2 L. Wang, Y. Ling, Y. Chen, C. L. Li, F. Feng, Q. D. You, N. Lu and Q. L. Guo, Flavonoid baicalein suppresses adhesion, migration and invasion of MDA-MB-231 human breast cancer cells, Cancer Lett., 2010, 297, 42-48.

3 L. L. Dong, Y. J. Fu, Y. G. Zu, M. Luo, W. Wang, X. J. Li and J. Li, Application of cavitation system to accelerate the endogenous enzymatic hydrolysis of baicalin and 
wogonoside in Radix Scutellariae, Food Chem., 2012, 131, 422-429.

4 Z. H. Gao, K. X. Huang, X. L. Yang and H. B. Xu, Free radical scavenging and antioxidant activities of flavonoids extracted from the radix of Scutellaria baicalensis Georgi, Biochim. Biophys. Acta, 1999, 1472, 643-650.

5 J. J. Liu, T. S. Huang, W. F. Cheng and F. J. Lu, Baicalein and baicalin are potent inhibitors of angiogenesis: inhibition of endothelial cell proliferation, migration and differentiation, Int. J. Cancer, 2003, 106, 559-565.

6 F. Zhou, Extraction of Total Flavonoids from Scutellaria baicalensis Georgi and Separation of Baicalin, Wuhan University of Engineering, Wuhan, 2007.

7 G. C. Chen, et al., Study on Microwave-assisted Extraction of Baicalin from Scutellaria baicalensis, J. Chem. Ind. Eng., 2005, 25, 81-83.

8 M. S. Featherand and J. F. Harris, The acid-catalyzed hydrolysis of glycopyranosides, Hydrolysis Glucopyranose, 1965, 30, 153-157.

9 B. Ismail and K. Hayes, Beta-glycosidase activity toward different glycosidic forms of isoflavones, J. Agric. Food Chem., 2005, 53, 4918-4924.

10 X. Wang, F. Wei, et al., Application of graphene oxide as a catalyst to accelerate extraction of total flavonoids and the hydrolysis of baicalin from Radix Scutellaria, Sep. Purif. Technol., 2014, 133, 421-428.

11 G. C. So and D. G. Macdonald, Kinetics of oil extraction from canola (rapeseed), Can. J. Chem. Eng., 1986, 64(1), 80-86.

12 D. C. Marcano, D. V. Kosynkin, D. C. Marcano, D. V. Kosynkin, J. M. Berlin, A. Sinitskii, Z. Z. Sun, A. Slesarev, B. L. Alemany, W. Lu and J. M. Tour, Improved synthesis of graphene oxide, ACS Nano, 2010, 4, 4806-4814.
13 Y. Wang, Z. Li, J. Wang, J. Li and Y. Lin, Graphene and graphene oxide: biofunctionalization and applications in biotechnology, Trends Biotechnol., 2011, 29, 205-212.

14 A. R. Linares, S. L. Hase, M. L. Vergara, et al., Modeling yerba mate aqueous extraction kinetics: Influence of temperature, J. Food Eng., 2010, 97(4), 471-477.

$15 \mathrm{~S}$. Mezuane and H. Kadi, Kinetics and thermodynamics of oil extraction from olive cake, J. Am. Oil Chem. Soc., 2008, 85(4), 391-396.

16 H. T. Zhu, C. Chao, L. Chen and Q. B. Wang, Study on extraction kinetics of total flavonoids from Prunella vulgaris, Journal of Chinese Pharmacists, 2012, 15(4), 459-461.

17 Y. C. Yang, M. C. Wei, T. C. Huang, S. Z. Lee and S. S. Lin, Comparison of modified ultrasound-assisted and traditional extraction methods for the extraction of baicalin and baicalein from Radix Scutellariae, Ind. Crops Prod., 2013, 45, 182-190.

18 L. L. Dong, Y. J. Fu, Y. G. Zu, J. Li, X. J. Li and T. Efferth, Negative pressure cavitation accelerated processing for extraction of main bioactive flavonoids from Radix Scutellariae, Chem. Eng. Process., 2011, 50, 780-789.

19 L. Feng, S. Zhang and Z. Liu, Graphene based gene transfection, Nanoscale, 2011, 3, 1252-1257.

20 S. F. Li and Z. Y. Jiang, Advanced Pharmaceutical Separation Engineering, Chemical Industry Press, Beijing, 2008, pp. 847.

21 J. S. Kang, Study on enzymatic extraction of Astragalus polysaccharides, Hefei University of Technology, Hefei, 2012.

$22 \mathrm{P}$. Zhao, Study on extraction and purification of polysaccharide from Coltsfoot, Northwestern Polytechnical University, Shaanxi, 2010.

23 X. D. He, S. H. Liu, G. L. Li, et al., Preparation of oxidized graphene and its catalytic hydrolysis of soybean isoflavones, J. Chem. Eng. Chin. Univ., 2012, 26(1), 56-60. 